
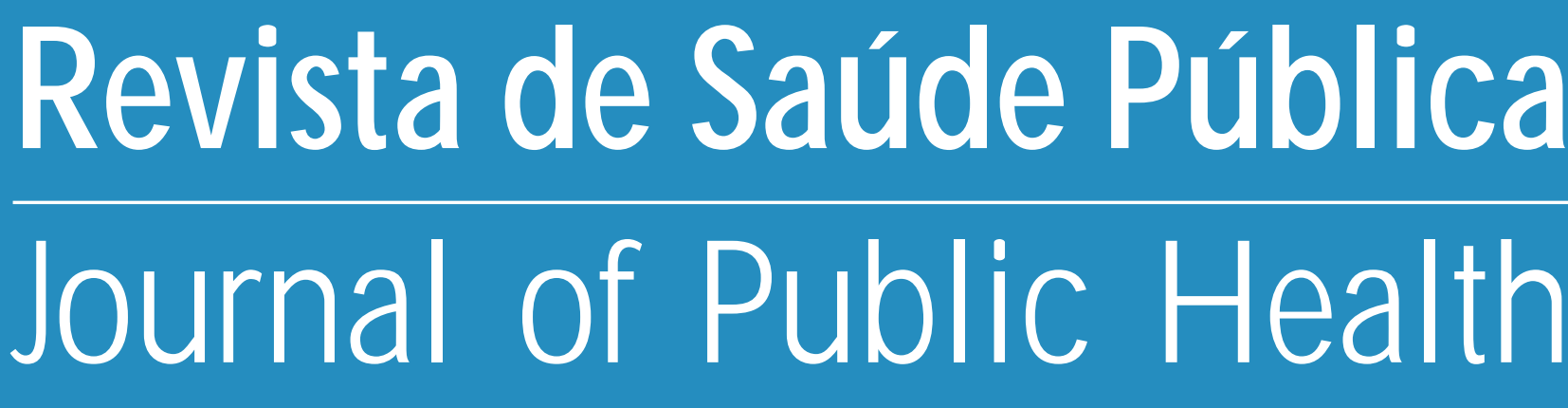

\title{
Modelos matemáticos de localização aplicados à organização espacial de unidades de saúde
} Mathematical location models applied in the spatial organization of health units

\section{Roberto D iéguez G alvão, Flávio Fonseca N obre e Miguel Murat Vasconcellos}

Coordenação dos Programas de Pós-G raduação em Engenharia da Universidade Federal do Rio de Janeiro. Rio de Janeiro, RJ - Brasil (RDG, FFN); Escola Nacional de Saúde Pública da Fundação Oswaldo Cruz. Rio 


\title{
Modelos matemáticos de localização aplicados à organização espacial de unidades de saúde* Mathematical location models applied in the spatial organization of health units
}

\author{
Roberto Diéguez Galvão, Flávio Fonseca Nobre e Miguel Murat Vasconcellos
}

Coordenação dos Programas de Pós-Graduação em Engenharia da Universidade Federal do Rio de Janeiro. Rio de Janeiro, RJ - Brasil (RDG, FFN ); Escola Nacional de Saúde Pública da Fundação O swaldo Cruz. Rio de Janeiro, RJ - Brasil (MMV)

\section{Descritores}

Serviços de saúde da mulher. Assistência perinatal.

Modelos teóricos.

\section{Keywords}

Women's health services.

Perinatal care.

Models, theoretical.

\section{Resumo}

Modelos matemáticos de localização têm tido aplicação crescente na área de saúde em nível internacional. No Brasil, embora de uso incipiente, existe enorme potencial para a utilização desses modelos na área de saúde pública. Nesse sentido são apresentados diversos modelos de localização com aplicação em saúde pública, analisando a localização de serviços não emergenciais, de serviços de emergência e a localização de serviços hierarquicamente relacionados. Mostrou-se a aplicação de um modelo hierárquico à localização de serviços de assistência materna e perinatal no Município do Rio de Janeiro, RJ (Brasil). Nesta parte, após a apresentação de alguns dados da assistência materna e perinatal no município, foi proposto um modelo hierárquico de quatro níveis (localização de unidades ambulatoriais, maternidades, centros de neonatologia e hospitais gerais) e analisado o impacto que a adoção da metodologia teria em comparação com o sistema atual.

\section{Abstract}

Mathematical location models have been increasingly applied in the health services at the international level. In Brazil, although incipient, there exists an enormous potential for the use of such models in the area of public health. In this paper several location models that can be applied to public health are presented initially, and the location of non-emergency services, of emergency services and of services hierarchically related are analysed. A hierarchical model is then applied to the location of maternal and perinatal assistance in the municipality of Rio de Janeiro. In this part, after presenting some related data for the municipality, a four-level hierarchical model (location of out-patient units, maternity hospitals, neonatal hospitals and general hospitals) is proposed and the impact that the adoption of this methodology would have as compared with that of the present system is analysed.
Correspondência para/Correspondence to: Flávio Fonseca Nobre, Caixa Postal 68510 21945-970 Rio de Janeiro, RJ - Brasil E-mail: flavio@peb.ufrj.br

\footnotetext{
* Baseado parcialmente na tese de doutorado, intitulada Modelos de localização e Sistemas de Informações Geográficas na assistência materna e perinatal: uma aplicação no município do Rio de Janeiro, 1997, apresentada à Coordenação dos Programas de Pós-Graduação em Engenharia da Universidade Federal do Rio de Janeiro (COPPE/UFRJ).

Subvencionado pela FINEP (Financiadora de Estudos e Projetos) e pelo CNPq (Conselho Nacional de Desenvolvimento Científico e Tecnológico)

Recebido em 17.3.1998. Reapresentado em 25.11.1998. Aprovado em 15.1.1999
} 


\section{INTRO DUÇÃO}

Nos últimos 25 anos as propostas de regionalização e hierarquização dos serviços de saúde no Brasil vêm sendo colocadas como necessárias para uma assistência de melhor qualidade a menor custo. Ao longo desse período foram elaboradas legislação detalhada e diversidade de normas com essa finalidade. Além disso, tiveram pouco êxito as tentativas de implementação de programas buscando regionalizar e hierarquizar os serviços de saúde segundo as normas e a legislação aprovadas.

Embora pouco utilizados no setor de saúde no Brasil, modelos matemáticos de localização de facilidades podem vir a ser uma ferramenta útil no planejamento de sistemas de saúde. Apesar das inevitáveis simplificações que devem ser feitas para representar um problema real através de um sistema de equações matemáticas, um modelo matemático pode ser considerado adequado se é capaz de prever com razoável precisão o efeito de mudanças em um dado sistema em sua "performance". Sua análise e solução podem fornecer informações valiosas sobre a operação do sistema ou organização em estudo.

$\mathrm{Na}$ construção de um modelo matemático deve ser definida uma medida de eficiência ou "performance" do sistema em estudo, conhecida como função-objetivo e expressa na forma $E=f\left(x_{i}, y_{i}\right)$, onde $E$ representa a eficiência do sistema, $x_{i}$ o conjunto de variáveis sujeitas a controle e $y_{i}$ o conjunto de variáveis não sujeitas a controle. As restrições aos valores que as variáveis podem assumir são expressas na forma de um conjunto de equações e/ou desigualdades que representam, por exemplo restrições operacionais do sistema em estudo.

O presente trabalho tem como objetivo apresentar uma introdução aos problemas de localização de facilidades e a modelos matemáticos que vêm sendo desenvolvidos para algumas das distintas categorias de problemas de localização, em especial para a localização de serviços não emergenciais e de serviços de emergência. Será também mostrada uma aplicação de um modelo hierárquico para a localização de serviços de assistência materna e perinatal no Município do Rio de Janeiro.

\section{PRO BLEMAS DE LO CALIZAÇÃO DE FACILIDADES}

O interesse em problemas de localização tem crescido bastante a partir da década de 60 , quando foram definidos os primeiros modelos matemáticos nessa área; a literatura correspondente é atualmente bastante vasta. O interesse presente concentra-se em modelos normativos (enfoque microeconômico), assim chamados porque caracterizam-se pela otimização de uma norma (medida de eficiência $E$ acima definida), sujeita às restrições operacionais relevantes. Os modelos normativos correspondem a problemas que podem ser formulados e resolvidos com base em técnicas de otimização de modelos matemáticos.

Problemas de localização podem ser classificados em três classes principais: (i) localização no plano com espaço infinito de soluções, (ii) localização no plano com espaço finito de soluções e (iii) localização em redes. Modelos de localização da classe (i) foram desenvolvidos a partir do trabalho de Alfred Weber ${ }^{25}$, que considerou a localização no plano de uma fábrica entre duas fontes de matéria-prima e um mercado consumidor. Esses modelos têm o inconveniente de poderem propor a localização de facilidades em lugares geograficamente pouco acessíveis ou mesmo inviáveis, como por exemplo no meio de um lago. Os modelos de localização em redes não possuem este inconveniente, já que a rede de interesse é definida pelos usuários do modelo, podendo os vértices por exemplo representar cidades e os arcos estradas de ligação entre as cidades.

ReVelle et al. ${ }^{19}$ analisaram modelos de localização sob o enfoque da propriedade das facilidades a serem localizadas: propriedade pública ou propriedade privada. Esses modelos diferem tanto pela formulação de objetivos e restrições, como principalmente pela natureza da propriedade. No setor privado o interesse maior é a minimização de custos ou maximização de lucros para os proprietários. No setor público o objetivo é a maximização de um benefício ou a minimização de um custo para a sociedade.

Problemas de localização no setor público podem ser classificados em duas categorias: localização de serviços não emergenciais e localização de serviços de emergência. Na primeira categoria estão incluídos a localização de escolas, de agências de correio, de edifícios públicos, de alguns serviços de saúde pública e mesmo de serviços relacionados ao meioambiente como suprimento de água e facilidades para o depósito de lixo. A categoria de serviços de emergência inclui por exemplo a localização de hospitais, de serviços de atendimento de emergência por ambulâncias e de estações do corpo de bombeiros. As medidas de eficiência a serem otimizadas são diferentes para as duas categorias. 
No caso de serviços não emergenciais uma medida de utilidade utilizada é a distância ou tempo médio de viagem dos usuários das facilidades a serem localizadas. Outra possível medida é a criação de demanda; neste caso a população usuária não é considerada fixa, mas uma função do número, tamanho e localização das facilidades. No caso de serviços de emergência uma medida bastante utilizada é a máxima distância a ser percorrida (ou tempo a ser gasto) entre qualquer usuário do sistema e a facilidade mais próxima.

As restrições de investimento podem tomar a forma de um limite superior no total a ser investido em construções, mais custos operacionais anuais, ou de um número específico de facilidades a serem operadas ${ }^{19}$. Uma questão importante que aparece nesses modelos é o horizonte de planejamento a ser utilizado. As facilidades devem ser localizadas para atender a demanda presente ou uma dada demanda futura? As facilidades devem ser expandidas na medida do necessário, ou devem ser construídas embutindo certa capacidade ociosa?

\section{MO DELOS PARA A LOCALIZAÇ̃̃O DE SERVIÇO S NÃO EMERG EN CIAIS}

Considere-se o seguinte modelo de localização. Dado um número de áreas de demanda por um certo produto ou serviço, cada uma com demanda conhecida, e um número de locais alternativos onde facilidades podem ser construídas para atender a demanda, determine-se o número de facilidades a serem construídas, onde cada facilidade deve ser localizada e que áreas de demanda devem ser atendidas por cada facilidade. O objetivo é atender totalmente a demanda, de modo que a soma dos custos de transporte com os custos amortizados de construir e operar as facilidades seja minimizada. Em termos de uma rede, as áreas de demanda e os locais alternativos para a construção de facilidades coincidem em geral com os vértices da rede, que representam áreas de concentração populacional.

No modelo em consideração existe claramente uma dependência entre os custos de transporte e os custos de construir e operar facilidades. Quanto maior o número de facilidades, menores os custos de transporte, mas maiores os custos relacionados à construção e operação das facilidades. O número de facili- dades a serem construídas representa o ponto ótimo de equilíbrio entre os dois custos que compõem a medida de utilidade a ser otimizada.

Em termos matemáticos o modelo assume a seguinte forma. Sejam:

$I \equiv J=\{1,2, \ldots, n\}-$ conjunto de vértices da rede em consideração;

$F_{i}\left(y_{i}, x_{i j}\right) \quad$ - custo de construir e operar uma facilidade em $i \in I$;

$\operatorname{dij}\left(x_{i j}\right) \quad$ - custo de suprir a demanda total da área $j \in J$ a partir de $i \in I$;

$x_{i j} \quad-$ fração da demanda da área $j \in J$ suprida a partir da facilidade $i \in I$;

$y_{i} \quad-$ variável discreta que assume apenas os valores 0 ou 1 :

$y_{i}=1$ se uma facilidade for construída em $i \in I$, $y_{i}=0$ caso contrário.

Então:

$$
\begin{array}{ll}
\text { Minimize } & Z=\sum_{i=1}^{n} F_{i}\left(y_{i}, \chi_{i j}\right)+\sum_{i=1}^{n} \sum_{j=1}^{n} d_{i j}\left(x_{i j}\right),(1) \\
\text { sujeito a } & \sum_{i=1}^{n} x_{i j}=1, j=1,2, \ldots, n \\
& x_{i j} \leq y_{i}, i=1, \ldots, n, j=1, \ldots, n, \\
& x_{i j} \geq 0, i=1, \ldots, n, j=1, \ldots, n, \\
& y_{i} \in\{0,1\}, i=1, \ldots, n .
\end{array}
$$

Se os custos de operar uma facilidade são diretamente proporcionais às quantidades despachadas a partir da facilidade, temos

$$
F_{i}\left(y_{i}, x_{i j}\right)=f_{i} y_{i}+\sum_{j=1}^{n} b_{i j} x_{i j},
$$

onde $f_{i}$ é o custo de se estabelecer uma facilidade em $i \in I$ e $b_{i j}$ é o custo de operar a demanda total da área $j \in J$ na facilidade $i \in I$. Partindo da hipótese que os custos de transporte são diretamente proporcionais às quantidades transportadas, temos

$$
d_{i j}\left(x_{i j}\right)=c_{i j} x_{i j},
$$

onde $c_{i j}$ é custo de suprir a demanda total da área $j \in J$ a partir da facilidade $i \in I$. Substituindo em (1) e simplificando obtém-se

$$
\begin{aligned}
& \text { Minimize } \quad Z=\sum_{i=1}^{n} f_{i} y_{i}+\sum_{i=1}^{n} \sum_{j=1}^{n}\left(b_{i j}+c_{i j}\right) x_{i j}, \mathrm{ou}, \\
& \text { fazendo } C_{i j}=b_{i j}+c_{i j}, \\
& \text { Minimize } \quad Z=\sum_{i=1}^{n} f_{i} y_{i}+\sum_{i=1}^{n} \sum_{j=1}^{n} C_{i j} x_{i j}
\end{aligned}
$$


sujeito a (2), (3), (4) e (5), que corresponde ao modelo de localização não capacitado, pelo fato de que não são impostos limites ao tamanho das facilidades.

$\mathrm{Na}$ formulação apresentada a função-objetivo busca minimizar os custos de construir e operar as facilidades, mais os custos de transporte do produto até as áreas de demanda. O número de facilidades a serem construídas resulta do equilíbrio ótimo entre os custos de construção e operação das facilidades e os custos de transporte, sendo determinado pelo número de variáveis $y_{i}=1$ na solução do problema. As restrições (2) estabelecem que a demanda de cada área deve ser totalmente atendida, ou seja, que a soma das frações da demanda suprida a partir do conjunto de todas as facilidades deve ser igual à unidade para cada área de demanda. As restrições (3) estabelecem que só é possível suprir áreas de demanda a partir de locais $i \in I$ onde existam facilidades, isto é, as variáveis $x_{i j}$ só podem assumir valores diferentes de zero se a variável $y_{i}$ correspondente for igual a 1 . Finalmente as restrições (4) e (5) estabelecem a natureza das variáveis do problema.

Note-se que (6) implica em que a função $F_{i}\left(y_{i}\right.$, $x_{i j}$ ) seja composta de um custo fixo $f_{i}$ mais um custo de operação linear, e que os custos de transporte são lineares, o que não é necessariamente verdadeiro, sendo portanto a expressão dada por (1) mais geral. A função $F_{i}\left(y_{i}, x_{i j}\right)$ é de forma geral não linear; os custos unitários de operação podem decrescer à medida que as quantidades manuseadas aumentam, em cujo caso estão presentes economias de escala. $\mathrm{Na}$ ausência desta, cada área de demanda é totalmente suprida a partir da facilidade mais próxima. Neste caso as variáveis $x_{i j}$ assumem apenas valores 0 ou 1 . Este é o caso da formulação dada pela função objetivo (6), sujeita às restrições (2), (3), (4) e (5); neste caso as restrições (4) podem ser substituídas por

$$
x_{i j} \in\{0,1\}, i=1, \ldots, n, j=1, \ldots, n .
$$

Para o setor público, um modelo bastante conhecido é denominado de problema das $\boldsymbol{p}$-medianas, no qual a função de utilidade a ser minimizada corresponde à distância total percorrida na rede, ou à distância média percorrida por um usuário do sistema para alcançar a facilidade mais próxima. No presente modelo o número de facilidades a serem operadas é fixado em $\boldsymbol{p}$. A restrição orçamentária pode ser expressa exclusivamente em termos de $\boldsymbol{p}$ quando o custo de construir e operar facilidades não varia entre pontos em potencial de localização das facilidades.
O modelo matemático toma a seguinte forma:

$$
\begin{array}{ll}
\text { Minimize } & Z=\sum_{i=1}^{n} \sum_{j=1}^{n} v_{j} d_{i j} x_{i j}, \\
\text { sujeito a } & \sum_{i=1}^{n} x_{i j}=1, j=1,2, \ldots, n, \\
& \sum_{i=1}^{n} y_{i}=p, \\
& x_{i j} \leq y_{i}, i=1, \ldots, n, j=1, \ldots n, \\
& x_{i j}, y_{i} \in\{0,1\}, i=1, \ldots, n, j=1, \ldots, n,
\end{array}
$$

onde $v_{j}$ é a população do vértice $j$ usuária em potencial do sistema (peso do nó $j$ ), $d_{i j}$ é a distância que corresponde ao caminho de comprimento mínimo entre os vértices $i$ e $j$ da rede e $\boldsymbol{p}$ é o número de facilidades a serem construídas e operadas no sistema.

Este modelo, que limita a função-objetivo à minimização de uma medida de utilidade social, com restrições que forçam o atendimento total da demanda dentro de um orçamento preestabelecido, tem tido ampla aplicação no setor público. Por exemplo, Pizzolato e Fraga da Silva ${ }^{18}$ utilizaram-no na localização de escolas no Município de Nova Iguaçu, no Rio de Janeiro; Vasconcellos ${ }^{24}$ na solução de um modelo hierárquico desenvolvido para a localização de unidades de atendimento materno e perinatal no município do Rio de Janeiro.

O modelo acima pode ser generalizado para incluir custos fixos na função-objetivo, mantendo uma restrição ao número máximo $\boldsymbol{p}$ de facilidades a serem operadas no sistema. Esta generalização é necessária quando o custo de construir e operar facilidades varia entre pontos em potencial de localização das mesmas. Neste caso, o modelo deve ser expresso em termos monetários, e a minimização da distância média percorrida por um usuário do sistema é substituída na função-objetivo pela minimização dos custos de transporte incorridos pelos usuários do sistema. $\mathrm{O}$ modelo neste caso é semelhante ao primeiro modelo mostrado nesta Seção, sendo definido matematicamente por:

$$
\begin{array}{ll}
\text { Minimize } & Z=\sum_{i=1}^{n} f_{i} y_{i}+\sum_{i=1}^{n} \sum_{j=1}^{n} c_{i j} x_{i j}, \\
\text { sujeito a } & \sum_{i=1}^{n} x_{i j}=1, j=1,2, \ldots, n, \\
& \sum_{i=1}^{n} x_{i} \leq p,
\end{array}
$$

e (10)-(11). 
Os custos $c_{i j}$ incluídos no duplo somatório da função-objetivo são apenas custos de transporte e a restrição (14) não está presente no modelo inicial. Assim, o número de facilidades a serem localizadas neste caso não representa necessariamente o ponto ótimo de equilíbrio entre os dois custos que compõem a medida de utilidade, pois está restringido a $\boldsymbol{p}$ facilidades por (14). Em outras palavras, o modelo acima incorpora uma restrição de investimento não considerada no modelo inicial. Por outro lado, é mais geral que o problema das $\boldsymbol{p}$-medianas, podendo ser utilizado tanto no setor público como no privado. $\mathrm{O}$ método das 3 Fases de Galvão e Raggi ${ }^{10}$ pode ser utilizado para obter a solução ótima desta formulação, que se convencionou chamar de modelo geral de localização não capacitado.

\section{A LO CALIZAÇÃO DE SERVIÇOS DE EMERG ÊN CIA}

Na localização de serviços de emergência buscase em geral prover cobertura a áreas de demanda. A noção de cobertura implica a definição de uma distância (tempo) de serviço, que é a distância (tempo) crítica além da qual a área de demanda é considerada não coberta. Uma área de demanda é, portanto, considerada coberta se está a menos da distância crítica de pelo menos uma das facilidades existentes, independentemente de a facilidade (ou servidor) estar ou não disponível quando o serviço é solicitado.

Considere-se por exemplo o problema de localizar serviços de atendimento de emergência por ambulâncias ou por estações do corpo de bombeiros em uma dada região, de tal modo que toda a população da região esteja a menos de 10 quilômetros de pelo menos uma das facilidades. Neste caso 10 quilômetros definem a distância crítica e o problema consiste na determinação do número mínimo de facilidades e de sua localização na região em consideração, de tal forma que cada área de demanda esteja a menos de 10 quilômetros de pelo menos uma das facilidades localizadas.

O mais simples dos modelos matemáticos existentes para problemas de localização com restrições de cobertura, que resolve o problema acima, é o Modelo de Localização para a Cobertura de Conjuntos (MLCC). Um problema a ele relacionado é o problema de localização dos $\boldsymbol{p}$-centros, que busca a localização de $\boldsymbol{p}$ facilidades de tal forma que a distância máxima de qualquer área de demanda à facilidade mais próxima seja a mínima possível.

Usualmente busca-se uma solução de compromisso que proporcione níveis de cobertura aceitáveis e seja financeiramente mais acessível às autoridades públicas. O Problema de Localização de Máxima Cobertura $(P L M C)^{4}$ foi desenvolvido com este propósito. Neste caso o objetivo é localizar um número pré-especificado de $\boldsymbol{p}$ facilidades, tal que a máxima população possível de uma dada região esteja coberta a menos de uma distância crítica $S$ pré-definida.

Matematicamente pode ser definido da seguinte forma. Seja $S$ a distância de serviço definida para o problema e sejam $J=\{1,2, \ldots, m\}$ o conjunto de áreas de demanda, $I=\{1,2, \ldots, n\}$ o conjunto de locais em potencial onde facilidades podem ser localizadas, $f_{j}$ a população da área de demanda $j, a_{i j}=1$ se a área de demanda $j$ puder ser coberta por uma facilidade localizada em $i \in I$ a menos da distância de serviço $S\left(a_{i j}=0\right.$ caso contrário), e $p$ o número de facilidades a serem localizadas. Convencione-se por outro lado $x_{j}=1$ se a área de demanda $j$ for coberta ( $x_{j}=0$ caso contrário); $y_{i}=1$ significa que uma facilidade deve ser localizada em $i \in I\left(y_{i}=0\right.$ caso contrário). A formulação matemática de PLMC é dada por:

$$
\begin{array}{ll}
\text { Maximize } & Z=\sum_{j \in J} f_{j} x_{j}, \\
\text { sujeito a } & \sum_{i \in I} a_{i j} y_{i}-x_{j} \geq 0, j \in J, \\
& \sum_{i \in I} y_{i}=p, \\
& x_{j} \in\{0,1\}, j \in J, \\
& y_{i} \in\{0,1\}, i \in I .
\end{array}
$$

$\mathrm{Na}$ formulação apresentada a função-objetivo busca maximizar a população total coberta. As restrições (16) asseguram que uma área de demanda $j \in J$ está coberta se existe pelo menos uma facilidade a menos da distância $S$ da mesma. A restrição (17) limita o número de facilidades na solução a $\boldsymbol{p}$. Finalmente, as restrições (17)-(18) definem a natureza binária das variáveis de decisão.

Do ponto de vista prático o modelo PLMC tem sido utilizado na solução de problemas reais em vários continentes, incluindo: a localização de centros de saúde em áreas rurais ${ }^{1}$; a localização de ambulâncias para atendimento de emergência em São Domingo, República Dominicana ${ }^{9}$; a localização de 
sirenes de aviso para casos de emergência ${ }^{6}$. Boffey e Narula $^{2}$ citam outras aplicações: localização de centros de saúde na Colômbia; análise de padrões de assentamentos arqueológicos no México; localização de poços artesianos e escolas em Benin, visando a erradicação da dracunculose; localização de equipamento para contenção e remoção de manchas de óleo em Long Island Sound (Estados Unidos). Métodos para a solução do problema são propostos, por exemplo, por Galvão e ReVelle ${ }^{11}$, Downs e Camm ${ }^{8}$, entre outros.

Uma desvantagem dos modelos apresentados na localização de serviços de emergência é que eles partem da hipótese que as facilidades (servidores) estão disponíveis quando solicitadas. Em sistemas não congestionados, com pouca demanda, a hipótese é razoável, mas em sistemas congestionados, nos quais chamadas freqüentes mantêm por exemplo ambulâncias na rua 80 a $90 \%$ do tempo, a hipótese é totalmente injustificada. $\mathrm{O}$ congestionamento em serviços de atendimento de emergência, que pode causar a não-disponibilidade de um servidor a menos da distância crítica quando solicitado, motivou o desenvolvimento dos modelos de localização com cobertura adicional ${ }^{13} \mathrm{e}$, posteriormente, dos modelos de cobertura probabilísticos ${ }^{7,20}$.

\section{MO DELOS DE LOCALIZAÇÃO HIERÁRQ UICOS}

Alguns sistemas que provêm produtos ou serviços relacionam-se hierarquicamente. Estes sistemas consistem em geral de $k(\geq 2)$ tipos distintos de facilidades. Por exemplo, sistemas de saúde podem consistir de clínicas e hospitais.

Narula ${ }^{16}$ propôs um esquema de classificação que leva em conta a hierarquia das facilidades (relação entre os vários tipos de facilidade), a disciplina do fluxo nos arcos e a disciplina do fluxo nos vértices. Norula define dois tipos de hierarquia entre facilidades: sucessivamente inclusiva e sucessivamente exclusiva. Em relação à primeira, uma facilidade oferece serviço a seu nível $e$ todos os serviços oferecidos por facilidades de hierarquia inferior à sua, em relação à segunda uma facilidade de um dado tipo oferece apenas serviços a seu nível (como por exemplo em sistemas telefônicos e de distribuição de energia).
Moore e ReVelle ${ }^{15}$ propuseram um modelo hierárquico sucessivamente inclusivo de dois níveis, que é uma extensão do PLMC, e o aplicaram à localização de facilidades para os serviços de saúde em Honduras. Nesse modelo as facilidades de hierarquia inferior (clínicas) oferecem apenas serviços de nível 1 , enquanto as facilidades de hierarquia superior (hospitais) oferecem tanto serviços de nível 2 quanto de nível 1 oferecidos pelas clínicas. Moore e ReVelle ${ }^{15}$ definiram distâncias críticas diferentes para os serviços de nível 1 e 2; além disso, a distância crítica para o serviço de nível 1 foi definida com valores diferentes para clínicas e hospitais. Modelos hierárquicos sucessivamente inclusivos foram também estudados por Calvo e Marks $^{3}$ e Schultz ${ }^{22}$, entre outros.

Em relação à disciplina de fluxo nos arcos, Narula $^{16}$ distingue entre fluxo integrado (fluxo de qualquer facilidade de hierarquia inferior para qualquer facilidade de hierarquia superior) e fluxo discriminado (fluxo de qualquer facilidade de hierarquia inferior $m$ para a facilidade de nível hierárquico imediatamente superior $m+1$ ). Finalmente, a disciplina de fluxo nos vértices pode incluir caminhos únicos ou caminhos múltiplos a partir de um dado vértice da rede.

Modelos hierárquicos podem ter como objetivo (i) minimizar a distância total ponderada percorrida até as facilidades (modelos minisoma), ou (ii) maximizar a população coberta a menos de uma distância de serviço crítica $^{15}$. Narula e $\mathrm{Ogbu}^{17}$ desenvolveram um modelo minisoma não capacitado de hierarquia sucessivamente inclusiva de 2 níveis, no qual $\boldsymbol{p}_{1}$ facilidades de hierarquia inferior (nível 1) e $\boldsymbol{p}_{2}$ facilidades de hierarquia superior (nível 2) devem ser localizadas entre $n\left(\geq p_{1}+p_{2}\right)$ vértices de uma rede. Nesse modelo uma fração $\theta(0 \leq \theta \leq 1)$ da demanda direcionada à facilidade de hierarquia inferior é referida à facilidade de hierarquia superior e no máximo uma facilidade pode ser localizada em um dado vértice.

\section{Modelo Hierárquico Minisoma de $\mathbf{N}$ arula e $\mathbf{O} \mathbf{g b u}$}

Além da notação descrita acima seja $W_{j}$ a demanda do vértice $j$ e $d_{j i}$ a distância mínima entre os vértices $j$ e $i$, as variáveis de decisão são:

$$
\begin{aligned}
X_{j i}^{01} \quad \text { - demanda do vértice } j \text { (no qual não exis- } & \text { te nenhuma facilidade), a qual é alocada } \\
& \text { a uma facilidade de nível } 1 \text { (hierarquia } \\
& \text { inferior) no vértice } i ;
\end{aligned}
$$


$X_{j i}^{02}-$ demanda do vértice $j$ (no qual não existe nenhuma facilidade), a qual é alocada a uma facilidade de nível 2 (hierarquia superior) no vértice $i$;

$X_{j j}^{01}\left(X_{j j}^{02}\right)$ - demanda do vértice $j$ que é alocada a uma facilidade de nível 1 (nível 2) existente neste vértice;

$X_{j i}^{12} \quad$ - demanda do vértice $j$ (no qual existe uma facilidade de nível 1), a qual é alocada a uma facilidade de nível 2 no vértice $i$;

$Y_{i}^{1} \quad=1$ se uma facilidade de nível 1 for localizada no vértice $i$ ( $=0$ caso contrário);

$Y_{i}^{2} \quad=1$ se uma facilidade de nível 2 for localizada no vértice $i$ ( $=0$ caso contrário).

O problema pode ser formulado matematicamente da seguinte forma:

$$
\begin{aligned}
\text { Minimize } & \sum_{i=1}^{n} \sum_{j=1}^{n}\left(X_{j i}^{01}+X_{j i}^{02}+X_{j i}^{12}\right) d_{j i}, \\
\text { sujeito a } & \sum_{i=1}^{n}\left(X_{j i}^{01}+X_{j i}^{02}\right)=W_{j}, j=1, \ldots, n, \\
& \sum_{i=1}^{n} X_{j i}^{12}=\theta \sum_{i=1}^{n} X_{i j}^{01}, j=1, \ldots, n, \\
& \sum_{j=1}^{n} X_{j i}^{01} \leq M Y_{i}^{1}, i=1, \ldots, n, \\
& \sum_{j=1}^{n} X_{j i}^{02}+\sum_{j=1}^{n} X_{j i}^{12} \leq M Y_{i}^{2}, i=1, \ldots, n, \\
& \sum_{i=1}^{n} Y_{i}^{1}=p_{1}, \\
& \sum_{i=1}^{n} Y_{i}^{2}=p_{2}, \\
& Y_{i}^{1}+Y_{i}^{2} \leq 1, i=1, \ldots, n, \\
& 0 \leq X_{j i}^{01} \leq M ; 0 \leq X_{j i}^{02} \leq M ; \\
& 0 \leq X_{j i}^{12} \leq \theta M, i, j=1, \ldots, n \\
& Y_{i}^{1}, Y_{i}^{2} \in\{0,1\}, i=1, \ldots, n,
\end{aligned}
$$

onde $M=\sum_{j=1}^{n} W_{j}$. Nessa formulação as restrições (21) asseguram que a demanda é totalmente atendida; as restrições (22) expressam o fato que uma fração $\theta$ da demanda alocada a facilidades de hierarquia mais baixa é referida a facilidades de hierarquia mais alta; as restrições (23) e (24) asseguram que alocações são feitas apenas a vértices onde facilidades estão localizadas; as restrições (25) e (26) indicam que serão localizadas $\boldsymbol{p}_{1}$ facilidades de nível 1 e $\boldsymbol{p}_{2}$ facilidades de nível 2; as restrições (27) garantem que em cada vértice será localizada no máximo uma facilidade. As restrições (28), embora redundantes, são úteis para uma maior clareza de exposição; finalmente, as restrições (29) definem a natureza binária das variáveis de decisão.

\section{Modelos Hierárquicos com Cobertura}

O modelo de Moore e ReVelle ${ }^{15}$ foi um dos primeiros modelos hierárquicos com cobertura publicados na literatura. Nesse modelo a cobertura é definida em termos de acesso a serviços e não em termos de acesso a facilidades. Uma área de demanda é portanto considerada coberta se tem acesso, a menos das distâncias críticas pré-definidas, tanto a serviços de nível 1 quanto a serviços de nível 2 na hierarquia de dois níveis. Como a hierarquia desse modelo é sucessivamente inclusiva, as facilidades de hierarquia inferior oferecem serviços de nível 1 , enquanto as de nível superior oferecem ambos os tipos de serviço.

O raciocínio que motivou a definição das distâncias críticas do modelo foi o seguinte. Seja $R_{l}$ a distância crítica para o nível 1 de serviços oferecidos pelas facilidades de hierarquia inferior. Em princípio essa distância crítica poderia ser considerada igual à distância crítica definida para o mesmo nível de serviços oferecidos pelas facilidades de hierarquia superior, mas na prática as pessoas podem dispor-se a viajar uma distância adicional para obter os mesmos serviços em uma facilidade com mais recursos. Assim $T_{1}$, a distância crítica para o nível 1 de serviços oferecidos pelas facilidades de hierarquia superior, deve satisfazer a $T_{1}>R_{1}$. Por outro lado, seja $R_{2}$ a distância crítica para o nível 2 de serviços. Esses serviços são oferecidos apenas pelas facilidades de hierarquia superior e na prática as pessoas podem mais uma vez dispor-se a viajar maiores distâncias para ter acesso aos serviços mais sofisticados de nível 2, de modo que nesse modelo considera-se $R_{2}>T_{1}>R_{1}$.

A formulação matemática do modelo de Moore e ReVelle ${ }^{15}$ não será aqui apresentada; detalhes da formulação e de métodos de solução para esse problema podem ser obtidos em Boffey et al.* Modelos hierárquicos de cobertura estão freqüentemente relacionados a serviços de emergência médica (SEM), os quais

* Trabalho inédito. Os leitores interessados poderão obter informações com os autores do presente artigo. 
consistem usualmente de unidades básicas de apoio (UBA) e unidades a vançadas de apoio (UAA). Os sistemas SEM são usualmente definidos em um contexto de modelos hierárquicos sucessivamente inclusivos. Eles são chamados de sistemas SEM de dois níveis (Mandell ${ }^{14}$ ). Tien et al. ${ }^{23}$ desenvolveram diversas formulações para o problema hierárquico de localização de unidades médicas. Mandell ${ }^{14}$ propôs um modelo que incorpora unidades UBAe UAA e considera a probabilidade que as demandas de cada vértice de uma rede sejam adequadamente atendidas para uma dada localização das unidades UBA e UAA.

\section{APLICAÇÃO NA LOCALIZAÇÃO DE SERVICCO S DE ASSISTÊN CIA MATERNA E PERINATAL NO MUNICÍPIO DO RIO DEJANEIRO}

O objetivo desta aplicação é mostrar como alguns dos modelos descritos no presente trabalho foram utilizados na localização hierarquizada dos serviços de assistência materna e perinatal no Município do Rio de Janeiro, e o impacto que sua aplicação teria na melhoria do atendimento da população do município. Objetivase também mostrar como o uso de SIG's, e a inserção dos modelos de localização hierarquizados nesse contexto, podem contribuir para uma melhor análise dos dados relevantes ao estudo, e para uma melhor compreensão, por parte das autoridades tomadoras de decisão, das diversas alternativas a sua disposição.

\section{A Assistência Materna e Perinatal no Município do Rio de Janeiro}

Os dados relativos à questão materna e perinatal do Município do Rio de Janeiro, estão detalhados em Vasconcellos $^{24}$. Não é objetivo do presente trabalho analisar esses dados, mas apenas fazer um sumário dos mesmos que justifique o sistema hierárquico utilizado.

O município tinha, em 1993, uma mortalidade perinatal de 19,50 por mil nascidos vivos, bem como os dados apontam também uma distribuição desigual da mortalidade no município, com índices mais elevados na Pavuna, Santa Cruz e São Cristóvão, e menos elevados em Copacabana e na Lagoa. Este risco desigual de morrer relacionado com o local de residência deve ser considerado em qualquer proposta de regionalização dos serviços perinatais, com melhoria do acesso espacial às tecnologias de assistência perinatais.
Os custos médios incorridos pelas gestantes para alcançar cada hospital, e os mesmos custos médios, agrupados por bairro, foram obtidos com base nas distâncias percorridas pelas gestantes até o hospital onde foi realizado o parto ${ }^{24}$. Os custos médios por hospital sugerem que valores mais baixos indicam localização mais adequada ou menor poder de atração; custos mais elevados sugerem o oposto: localização menos adequada e/ou maior poder de atração. Já a análise desses custos, segundo o bairro de residência, aponta para uma grande diferença entre os bairros, sugerindo forte desigualdade do acesso espacial a serviços de assistência ao parto.

Em conclusão, as análises de Vasconcellos ${ }^{24}$ apontam o setor público como responsável pelo financiamento de $70 \%$ dos nascimentos no município, assim como uma distribuição espacialmente desigual da mortalidade neonatal, sugerindo a viabilidade e a necessidade da intervenção por parte do poder público. Há assim um forte incentivo a estudos de planejamento dos serviços de assistência materna e perinatal no Município do Rio de Janeiro, com base em modelos matemáticos de localização, de forma regionalizada e hierarquizada. Regionalizada no sentido de uma melhor distribuição espacial dos serviços ofertados, e hierarquizada no sentido de melhor organização e menores custos na prestação dos serviços. Nesse sentido as facilidades de hierarquia superior devem concentrar os equipamentos e procedimentos mais sofisticados, tendo sua demanda filtrada pelas de hierarquia inferior, de modo a obter-se melhor desempenho econômico e operacional do sistema.

\section{O Sistema Hierárquico Proposto}

Definida como diferenciação da capacidade tecnológica das unidades, em forma de crescente complexidade, a hierarquização está em nosso caso associada ao conceito de risco ou de necessidade. $\mathrm{Na}$ assistência materna e perinatal tanto a mãe como o recém-nascido podem ser classificados em diferentes graus de risco, segundo determinados critérios clínicos. O risco do recém-nascido foi definido como baixo, médio e alto; o risco da mãe foi classificado em baixo e alto (por exemplo: diabéticas, cardiopatas, nefropatas e aidéticas $)^{24}$.

O esquema de hierarquia utilizado foi o de hierarquia sucessivamente inclusiva ${ }^{16}$. O sistema de referência, considerando tanto o risco da mãe quanto o do recém-nascido, começa no período pré-natal (ges- 
tação), continua durante o parto e termina no período neonatal. De forma a contemplar essa diversidade de possibilidades define-se nível de assistência como a capacidade tecnológica para atender cada uma das situações de risco. Foram assim definidos quatro tipos de Unidades:

- Ambulatório - deve possuir tecnologia compatível com assistência pré-natal e recém-nascidos de baixo risco. Trata-se do nível I no sistema hierárquico;

- Maternidade - deve possuir a capacidade tecnológica do nível I, acrescida daquela para a assistência pré-natal a recém-nascidos de médio risco. É o nível II da hierarquia. As maternidades devem também prover o atendimento ao parto e período pósparto de mães e recém-nascidos de baixo e médio risco;

- Centro de Neonatologia - deve possuir a capacidade tecnológica dos dois níveis anteriores, acrescida daquela compatível com atendimento de recém-nascidos de alto risco, incluindo o pré-natal (nível III da hierarquia);

- Hospital Geral - deve possuir a capacidade tecnológica dos níveis anteriores, acrescida da capacidade de atender mães de alto risco, incluindo o prénatal. Trata-se do nível IV da hierarquia.

O número de unidades necessárias em cada nível da hierarquia, partindo do princípio de um atendimento adequado no Município do Rio de Janeiro, foi obtido usando-se o modelo de programação de ações de saúde de Rivera ${ }^{21}$, bastante difundido no setor de saúde, com utilização de parâmetros propostos pelo Comitê da Assistência Médica Reprodutiva de Ontário e pela Comissão Perinatal do Município do Rio de Janeiro5.

O esquema utilizado para a localização das unidades dos diferentes níveis hierárquicos foi o seguinte. Os ambulatórios (nível I) foram localizados utilizando-se o modelo correspondente ao problema das $\boldsymbol{p}$-medianas, em uma rede constituída pelos centróides de cada um dos bairros do Município do Rio de Janeiro (os vértices da rede), conectados entre si pelas principais vias de tráfego (os arcos da rede). O modelo das $\boldsymbol{p}$-medianas localiza as $\boldsymbol{p}$ unidades de tal forma que as distâncias de acesso às unidades, por parte da população usuária, sejam mínimas. Além da localização "ótima" das unidades, que como provou Hakimi ${ }^{12}$ ocorre sempre nos vértices da rede, a solução inclui a vinculação de cada um dos bairros às unidades localizadas. Como população de cada bairro (peso $v_{j}$ da função objetivo (7) foi utilizado o número de nascidos vivos no bairro. A rede foi portanto ponderada pelos nascimentos no bairro de residência da mãe.

$\mathrm{Na}$ localização das maternidades e centros de neonatologia (níveis II e III do sistema hierárquico proposto) foi utilizado o modelo hierárquico minisoma de Narula e $\mathrm{Ogbu}^{17}$, mencionado anteriormente, em uma rede onde os vértices são os ambulatórios localizados na etapa anterior, conectados entre si pelas principais vias de tráfego. Como além da minimização das distâncias percorridas objetivou-se $o$ atendimento dos recém-nascidos de risco nos centros mais próximos, essa metodologia pareceu a mais adequada. Além de minimizar a distância média percorrida, ela leva em conta o percentual de gestantes/ recém-nascidos referidos ao nível III da hierarquia, fornecendo além disso a localização de maternidades e centros de neonatologia de forma integrada.

A metodologia acima assegura o esquema de referência dos ambulatórios para as maternidades, embora de forma não necessariamente ótima do ponto de vista matemático. Ela também assegura a referência de mães com feto/bebê de alto risco e de bebês de alto risco para os centros de neonatologia de forma (matematicamente) ótima. Como demanda (peso $v_{i}$ ) associada a cada ambulatório localizado em um vértice $i$ da rede foi utilizada a soma do número de nascidos vivos dos bairros vinculados ao ambulatório localizado em $i$.

Finalmente, na localização dos hospitais gerais voltou a ser utilizado o modelo das $\boldsymbol{p}$-medianas, em uma rede cujos vértices são os centros de neonatologia, localizados segundo a metodologia já descrita, sendo os arcos constituídos pelas principais vias de tráfego da rede.

A construção da rede básica, constituída pelos centróides dos bairros (vértices), articulados entre si pelas principais vias de tráfego, teve como base dois mapas digitalizados usando-se o AutoCad ${ }^{\circledR}$ e, posteriormente o ArcInfo®, para geração de topologia e correções necessárias. $\mathrm{O}$ primeiro corresponde a malha de setores censitários do Censo Demográfico de 1991, e foi gerado pelo projeto SIG/FIOCRUZ, da Fundação Osvaldo Cruz, na escala 1:5.000. Os bairros e regiões administrativas foram obtidas por agregação dos setores.

O segundo mapa utilizado, na escala 1:60.000, foi cedido pelo Programa de Engenharia de Transportes da Coordenação dos Programas de Pós-gra- 
duação em Engenharia (COPPE). Esse mapa contém as rodovias federais, estaduais, municipais e as ruas, bem como rodovias planejadas na época de sua execução, incluindo as linhas vermelha e amarela.

O processamento de dados foi realizado em estação de trabalho digital 3.000/300 do Programa de Engenharia de Produção da COPPE.

\section{Comparação com o Sistema Atual}

O sistema atual (dados de 1995) é mostrado nas Figuras 1 e 2. Na Figura 1 são mostrados os estabelecimentos ambulatoriais públicos com capacidade para atendimento a gestantes. Na Figura 2 são mostradas as maternidades, centros de neonatologia e hospitais gerais que realizaram procedimentos obstétricos financiados pelo SUS (Sistema Único de Saúde) em 1995.

Vasconcellos ${ }^{24}$ aplicou o modelo hierárquico proposto a dois diferentes cenários, levando em conta as recomendações da Comissão Perinatal do Município do Rio de Janeiro ${ }^{6}$ e tendo como base 100.000 nascidos vivos anuais no município, um número de abortamentos (espontâneos e provocados) de $20 \%$ e uma cobertura de $80 \%$ das gestações. No cenário I ele sugere a meta de atender 100.000 gestantes anuais (supondo existência de demanda reprimida) e no cenário II (mais conservador) a meta de atender 80.000 gestantes anuais.

Apresentam-se os resultados relativos ao cenário I que aponta a necessidade de 69 unidades ambulatoriais: 23 maternidades, 7 centros de neonatologia e 2 hospitais gerais, para atendimento materno e perinatal no Município do Rio de Janeiro. Na Figura 3 é mostrada a localização das 69 unidades ambulatoriais, obtida a partir do modelo das $\boldsymbol{p}$-medianas, conforme descrito em 6,2 acima. As 69 unidades ambulatoriais foram localizadas nos centróides de bairros; bairros sem unidades ambulatoriais localizadas em seu centróide têm o atendimento de suas gestantes alocado a ambulatórios localizados em bairros próximos.

Na Figura 4 é mostrada a localização das 23 maternidades, 7 centros de neonatologia e 2 hospitais gerais. Conforme descrito anteriormente, as maternidades e os centros de neonatologia foram localizados de forma integrada, tendo sido utilizado o modelo hierárquico minisoma de Narula e Ogbu ${ }^{17}$. Já os hospitais gerais foram localizados utilizando-se o modelo das $\boldsymbol{p}$-medianas.

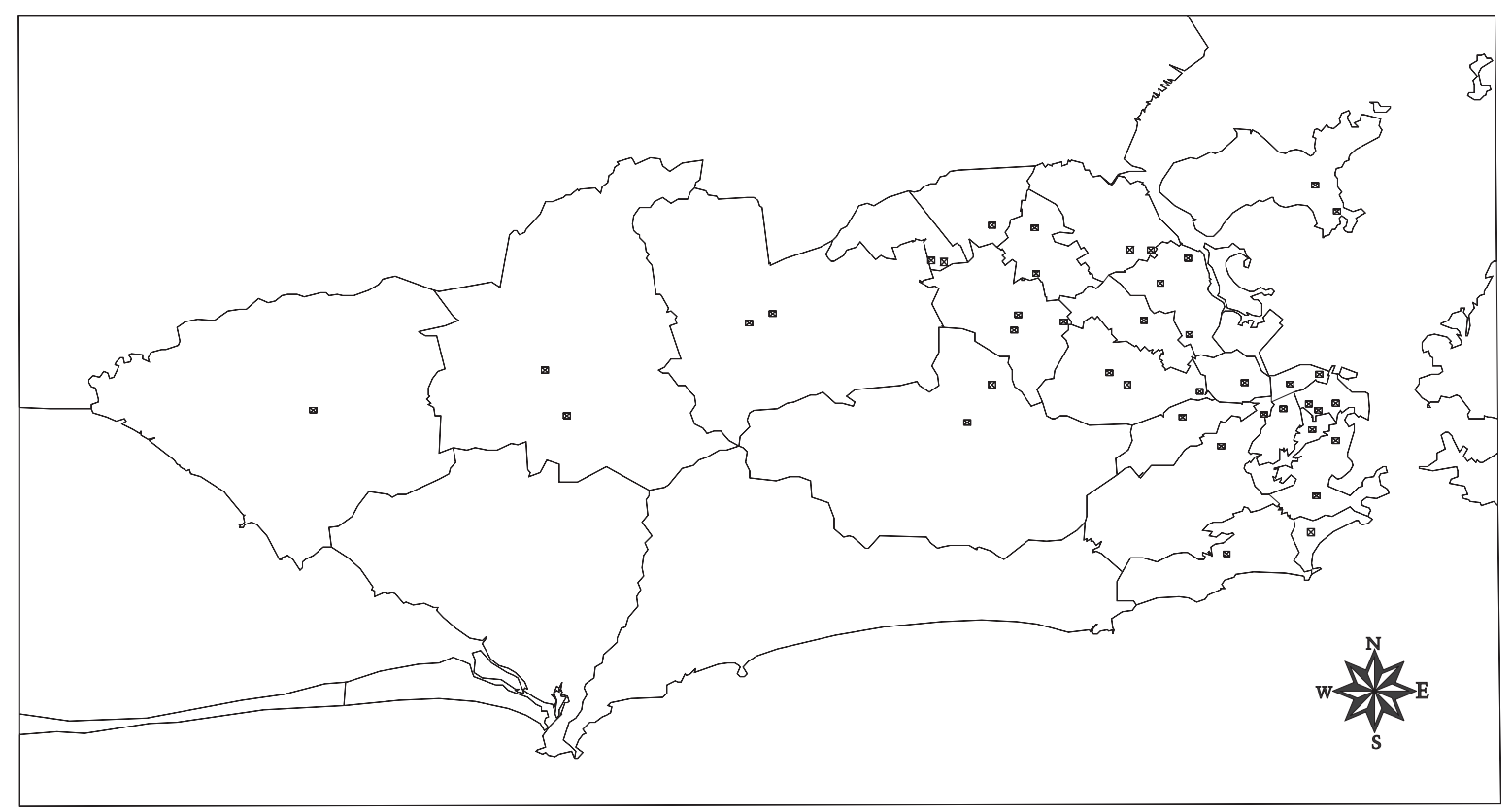

^ Centros de saúde e postos de assistência médica existentes

- Limites de região administrativa

Figura 1 - Distribuição geográfica dos estabelecimentos ambulatoriais públicos com capacidade para atendimento à gestantes em 1995. 


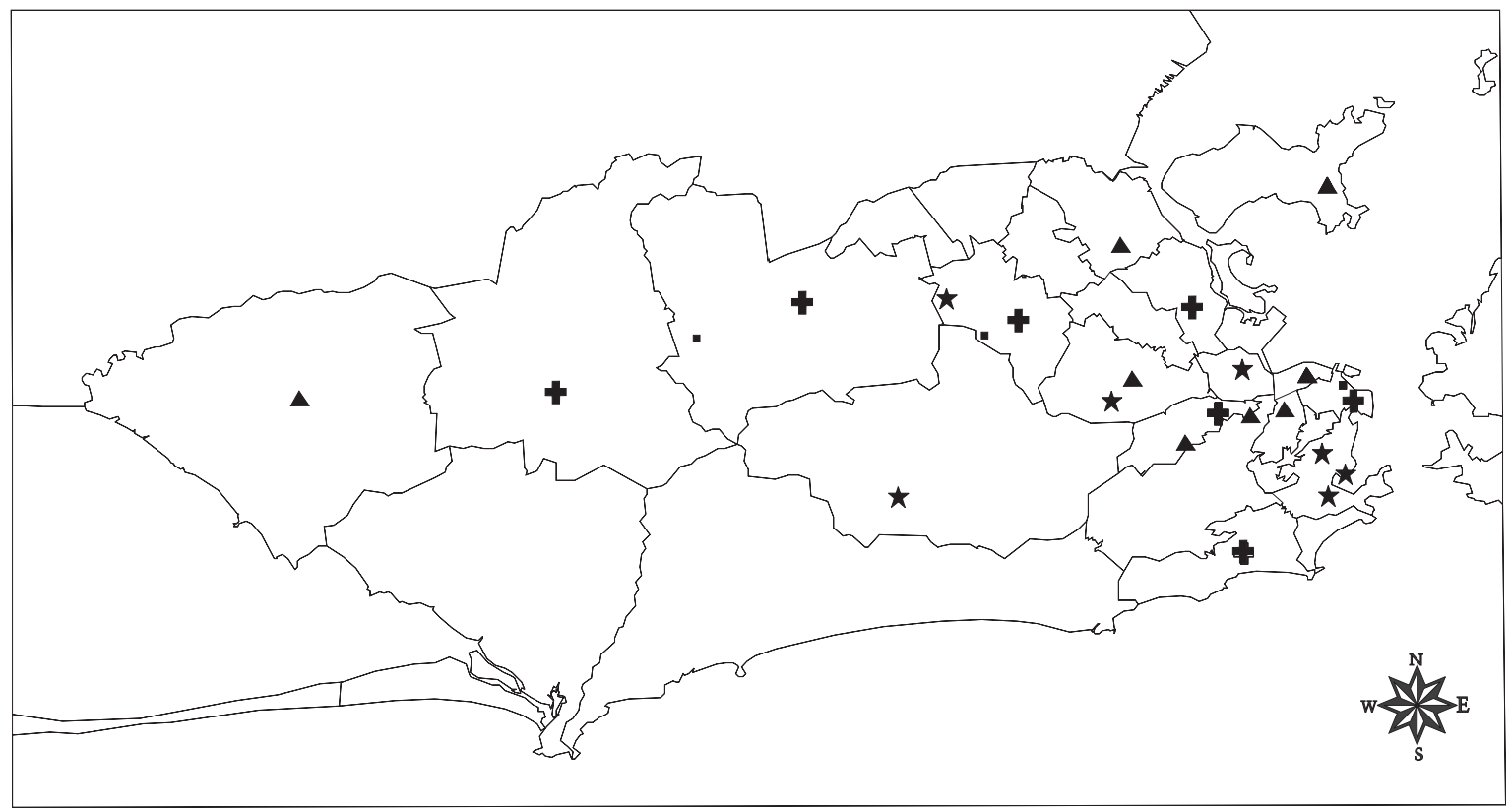

- Maternidade

- Maternidade em Hospital Geral

$\star$ Centro de Neonatologia em Maternidade

+ Maternid. e Centro de Neonat. em Hosp. Geral

Figura 2 - M aternidades, centros de neonatologia e hospitais gerais que realizaram procedimentos obstétricos financiados pelo Sistema Ú nico de Saúde em 1995.

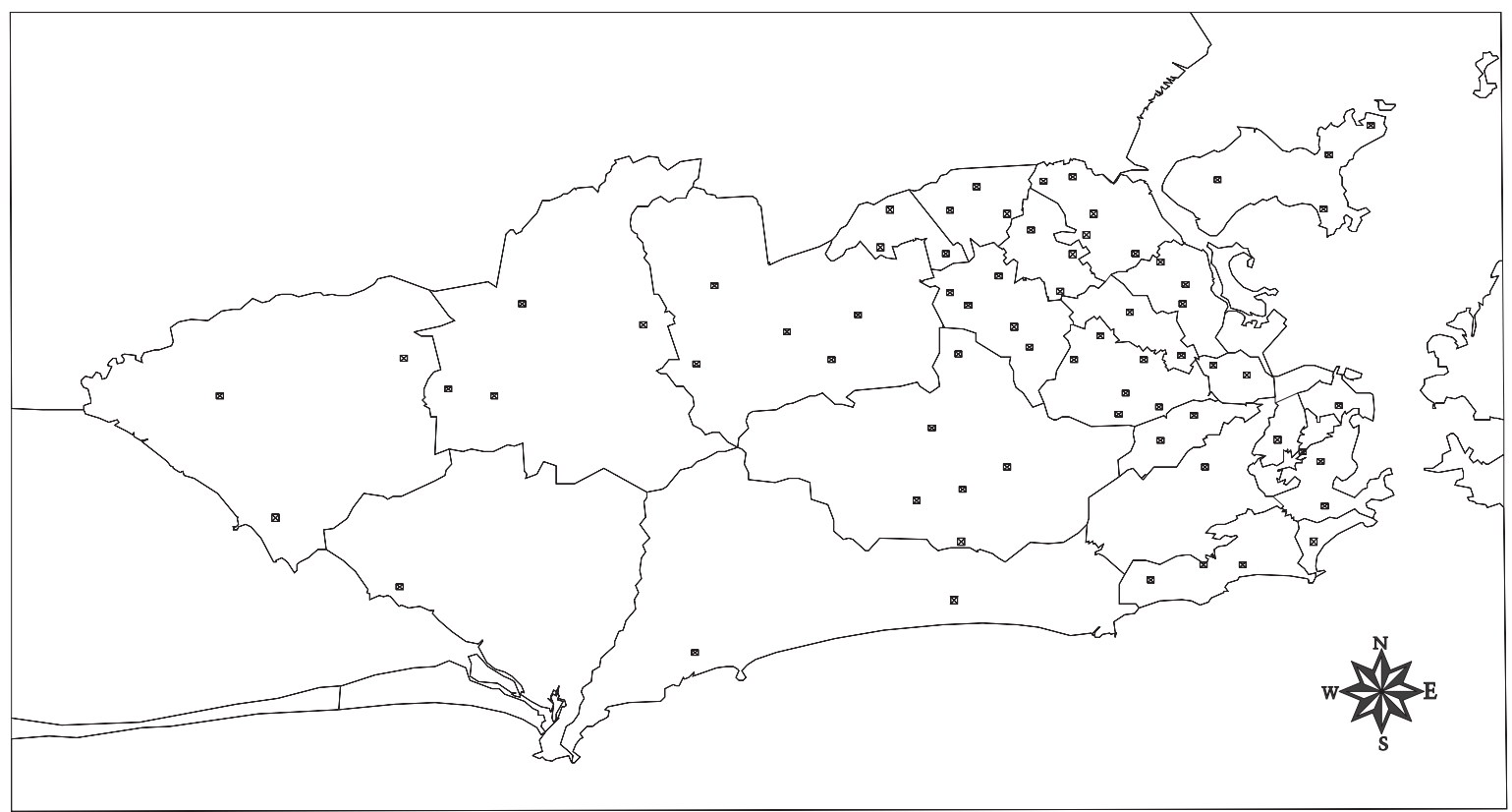

凶 Localização de ambulatório

- Limites de região administrativa

Figura 3 - Localização geográfica das unidades ambulatoriais para o cenário I. 


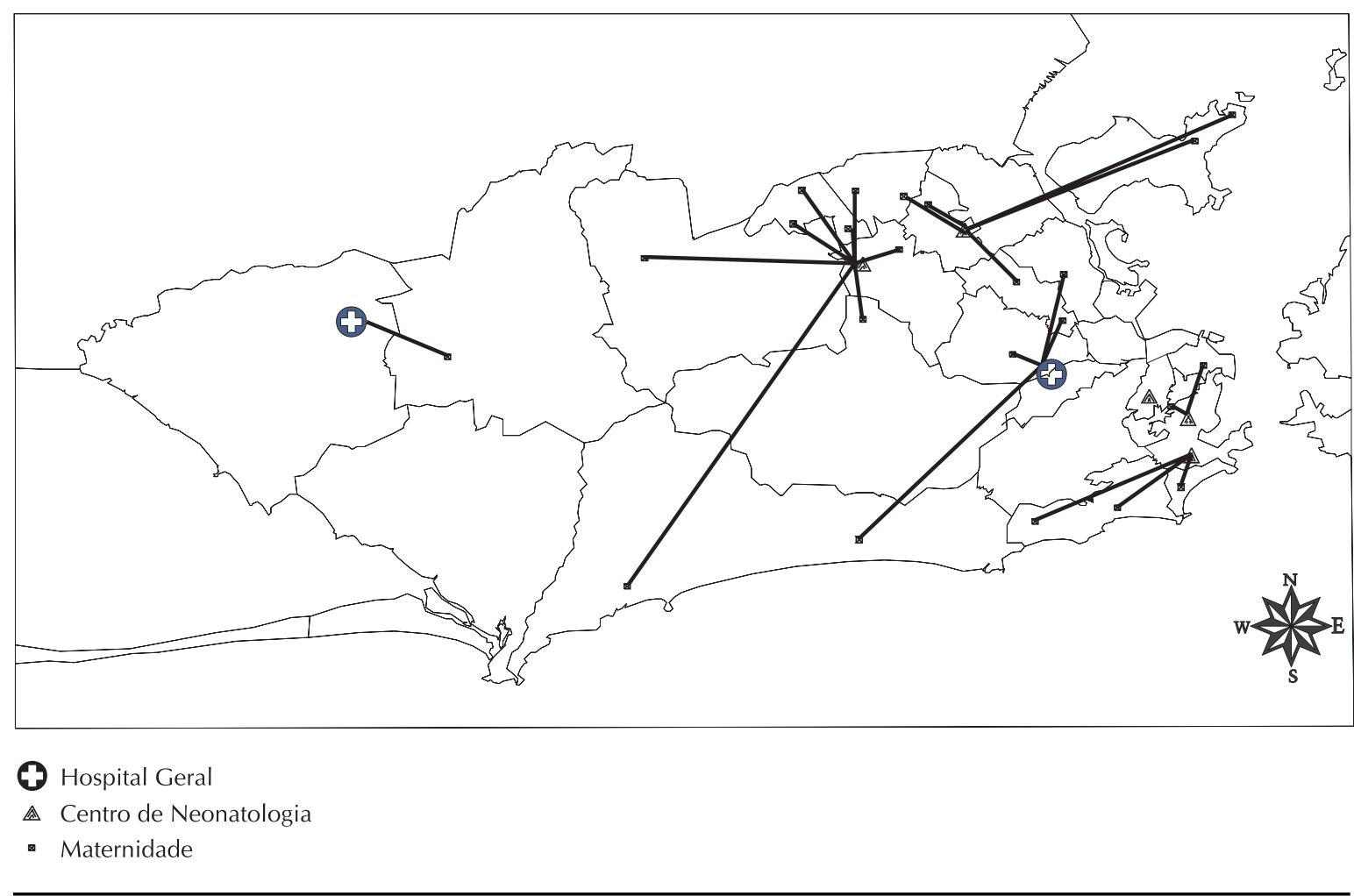

Figura 4 - Localização de maternidades, centros de neonatologia e hospitais gerais, para o cenário I.

A comparação das Figuras 1 e 2 com as Figuras 3 e 4 mostra, de forma espacial, a melhor configuração de um atendimento planejado através de modelos de localização. Alguns números reforçam esta impressão visual. Por exemplo, a distância média ponderada percorrida por gestantes no sistema atual (dados de 1995), entre o bairro de sua residência e o bairro de ocorrência do parto, em hospitais financiados pelo SUS, foi de aproximadamente 11 quilômetros. Já se a localização proposta estivesse implementada esta distância média cairia para menos de 2 quilômetros. Uma discussão mais detalhada do assunto, incluindo uma análise das regiões administrativas mais e menos favorecidas no sistema atual, é feita em Vasconcellos ${ }^{24}$.

\section{CONCLUSÕES}

Modelos de localização podem constituir-se importante ferramenta de apoio à organização dos serviços de saúde no Brasil. Isto ganha maior dimensão ao constatar-se que apesar da abundante legislação e grande quantidade de normas visando a regionalizar e hierarquizar a rede de serviços de saúde, pouco foi implementado até o presente. Em relação ao atendimento materno e perinatal no Município do Rio de Janeiro, a situação de crise atual, com elevado número de óbitos em unidades de atendimento neonatal, a partir de janeiro de 1998, torna óbvia a necessidade de maiores investimentos e de um melhor planejamento desse setor.

As possibilidades redistributivas implícitas nos modelos de localização e as vantagens de sua integração a Sistemas de Informações Geográficas podem e devem ser utilizadas no planejamento da saúde pública no Brasil. Adelimitação de áreas com populações específicas e homogêneas, aliada à vinculação dessas áreas a estruturas de serviços de saúde, permite visualizar prioridades e apoiar decisões relacionadas à localização de serviços de saúde e de tecnologia, articulados em estruturas regionalizadas e hierarquizadas. 


\section{REFERÊNCIAS}

1. Bennett VL, Eaton D, Church RL. Selecting sites for rural health workers. Soc Sci Med 1982; 16:63-72.

2. Boffey TB, Narula SC. Multiobjective covering and routing problems. In: Karwan MH, Spronk J, Wallenius J editors. Essays in decision making: a volume in honor of Stanley Zionts. Berlin: Springer-Verlag; 1997. p. 342-69.

3. Calvo AB, Marks DH. Location of health care facilities: an analytic approach. Socio-econ Plann Sci 1973; 7:407-22.

4. Church R, Revelle CS. The maximal covering location problem. Pap Reg SciAssoc 1974; 32:101-18, .

5. Comisão Perinatal do Município do Rio de Janeiro. Plano de regionalização e hierarquização da atenção pré-natal e perinatal. Rio de Janeiro, 1987.

6. Current J, O'Kelly M. Locating emergency warning sirens. Decisi Sci 1992; 23:221-34.

7. Daskin MS. Application of an expected covering model to emergency medical service system design. Decis Sci 1982; $13: 416-39$

8. Downs BT, Camm JD. An exact algorithm for the maximal covering location problem. Nav Res Logistics Q 1996; 43:435-61.

9. Eaton D, Hector M, Sanchez V, Latingua R, Morgan J. Determining ambulance deployment in Santo Domingo, Dominican Republic. J Oper Res Soc 1986; 37:113-26.

10. Galvão RD, Raggi LA. A method for solving to optimality uncapacitated location problems. Annals Oper Res 1989; 18:225-44

11. Galvão RD, Revelle CS. A Lagrangean heuristic for the maximal covering location problem. Eur J Oper Res 1996; $88: 114-23$

12. Hakimi SL. Optimum distribution of switching centers in a communication network and some related graph theoretic problems. Oper Res 1965; 13:462-75.
13. Hogan K, Revelle CS. Concepts and applications of backup coverage. Manage Sci 1986; 32:1434-44.

14. Mandell MB. Covering models for two-tiered emergency medical services systems. In: 7th International Symposium on Locational Decisions - ISOLDE VII 1996; Alberta (Ca) v. 9 , p. 79.

15. Moore GC, Revelle CS. The hierarchical service location problem. Manage Sci 1982; 28:775-80.

16. Narula SC. Hierarchical location-allocation problems: a classification scheme. Eur J Oper Res 1984; 15:93-9.

17. Narula SC, Ogbu UI. Lagrangean relaxation and decomposition in an uncapacitated 2-hierarchical location-allocation problem. Comput Oper Res 1985; 12:169-80.

17. Pizzolato ND, Fraga da Silva HB. The location of public schools: evaluation of practical experiences. Int Trans Oper Res 1997; 4:13-22.

19. Revelle CS, Marks D, Liebman JC. An analysis of private and public sector location models. Manage Sci 1970; 16:692-707.

20. Revelle CS, Hogan K. The maximum availability location problem. Transport Sci 1989; 23: 192-200.

21. Rivera RJU. Programação local de saúde, distritos sanitários e enfoque estratégico. In: Rivera, FJU editor. Planejamento e programação em saúde: um enfoque estratégico. São Paulo: Cortez/Abrasco; 1989. p. 177-202.

22. Schultz GP. The logic of health care facility planning. Socioecon Plann Sci 1970; 4:383-93.

23. Tien JM, El-Tell GR, Simons GR. Improved formulations to the hierarchical health facility location-allocation problem. IEEE Trans Syst Man Cybern 1983; 13:1128-32.

24. Vasconcellos MM. Modelos de localização e sistemas de informações geográficas na assistência materna e perinatal: uma aplicação no Município do Rio de Janeiro. Rio de Janeiro; 1997. [Tese de doutorado - Coordenação dos Programas de Pós-graduação em Engenharia da UFRJ].

25. Weber A. Alfred Weber's theory of the location of industries Chicago (Ill): University of Chicago Press; 1929. 